\title{
Early Complications in Patients Undergoing Elective Open Surgery for Infrarenal Aortic Aneurysms
}

\author{
Dariusz Janczak1, Wojciech Bakowski2, Katarzyna Bakowska2, Karol Marschollek2, Pawel Marschollek2, \\ Maciej Malinowski1 and Mariusz Chabowski2,3 \\ ${ }^{1}$ Department of Vascular, General and Transplantation Surgery, Faculty of Postgraduate Medical Training, Wroclaw Medical University, \\ Wroclaw, Poland \\ ${ }^{2}$ Division of Surgical Specialties, Department of Clinical Nursing, Faculty of Health Science, Wroclaw Medical University, \\ Wroclaw, Poland \\ ${ }^{3}$ Department of Surgery, $4^{\text {th }}$ Military Teaching Hospital, Wroclaw, Poland
}

\begin{abstract}
Objective: To assess the type and frequency of early postoperative complications in patients after open surgical infrarenal aneurysms repair and determination of their risk factors.

Study Design: An observational study

Place and Duration of Study: The 4th Military Teaching Hospital in Wroclaw, Poland, from January 2012 to December 2016. Methodology: Analyses of medical records of 205 patients treated for infrarenal aneurysm with open surgery. Patients with clinical and radiological features of aneurysm rupture were excluded. Complications and early deaths ( $\leq 30$ days) were recorded.

Results: Men represented $170(83 \%)$ of patients, the average age was $67.9 \pm 6.68$ years. Aneurysm diameter $<60 \mathrm{~mm}$ occurred in $107(52.2 \%)$ of patients, $60-80 \mathrm{~mm}$ in $76(37.1 \%)$, and $>80 \mathrm{~mm}$ in $22(10.7 \%)$. Aortic prostheses (tube grafts) were implanted in $132(64.4 \%)$ of patients and aorto-bifemoral (bifurcated) grafts in 73 (35.6\%). The most common postoperative complications were: reoperation necessity $(n=23 ; 11.2 \%)$, respiratory failure $(n=21 ; 10.2 \%)$, respiratory tract infection $(n=14 ; 6.8 \%)$, circulatory failure $(n=14 ; 6.8 \%)$, lower limb ischemia $(n=13 ; 6.3 \%)$, kidney failure $(n=12 ; 5.9 \%)$, death $(n=9 ; 4.4 \%)$, and surgical wound infection $(n=9 ; 4.4 \%)$. Statistical analysis indicated intraoperative blood loss, duration of surgery, a larger aneurysm diameter, and diabetes as risk factors for early complications.

Conclusion: Complications were the same as reported from other centres. There is a need to shorten surgery duration and minimise blood loss, as these modifiable factors were the predictors of early complications.
\end{abstract}

Key Words: Aortic aneurysm, Abdominal surgery, Postoperative complications

How to cite this article: Janczak D, Bakowski W, Bakowska K, Marschollek K, Marschollek P, Malinowski M, Chabowski M. Early complications in patients undergoing elective open surgery for infrarenal aortic aneurysms. J Coll Physicians Surg Pak 2019; 29(11):1078-82.

\section{INTRODUCTION}

Abdominal aortic aneurysm (AAA) is a pathological dilation of the aorta with its diameter $50 \%$ greater than normal value (about $3 \mathrm{~cm}$ in the most frequent localisation - under the renal arteries) with the loss of parallelism of its walls. ${ }^{1}$ The incidence of AAA ranges from $4 \%$ to $8 \% 2,3$ and it is more frequent than thoracic aorta aneurysms (TAA). ${ }^{4}$ In more than $25 \%$ of patients, co-occurrence of aneurysms of both above-mentioned locations is observed, which significantly complicates its management. 4,5 The most important factors for AAA development include advanced age, male gender, positive family history, smoking, presence of other large

Correspondence to: Mariusz Chabowski, Department of Surgery, 4th Military Teaching Hospital, 5 Weigla Street, 50-981 Wroclaw, Poland.

E-mail: mariusz.chabowski@gmail.com

Received: March 25, 2019; Revised: September 05, 2019;

Accepted: September 05, 2019 aneurysms or atherosclerosis. ${ }^{4-7}$ Genetic predispositions include Ehlers-Danlos 8, Turner and Marfan syndromes. 8,9

Abdominal aortic aneurysms tend to constantly expand their lumen. The speed of expansion depends on the size - large aneurysms usually develop faster than small ones. 6,7 The strongest risk factor for the rapid increase of an aneurysm diameter is smoking. ${ }^{1}$ There are also factors that delay the development of an aneurysm. It was noticed that in patients with diabetes, the progression of the aneurysm enlargement is noticeably smaller, and the same group has a reduced risk of AAA. The reasons for such a relationship remain unknown. ${ }^{10}$

In most cases, AAA does not give any symptoms before rupture. ${ }^{1}$ Only some of them may cause abdominal pain radiating to the back or groin. ${ }^{11}$ Abdominal palpation may reveal a pulsating mass, but the sensitivity of this examination is low. ${ }^{1}$ This leads to a situation, in which many patients with AAA remain undiagnosed.

The conservative treatment of this disease is based on the administration of antihypertensive drugs such as 
beta-blockers and ACE inhibitors, which reduces the risk of rupture of the aneurysm. ${ }^{1}$ The benefits of using statins have also been demonstrated. 12

There are two methods of surgical treatment: classic open aneurysm repair (OAR) introduced in the 1950s and endovascular aneurysm repair (EVAR), which has been used since 1990s. There are few publications on this topic in the world literature.

Open surgical repair of AAA is associated with significantly high morbidity and mortality. For infrarenal AAA, 30 -days mortality was reported to be $1,5 \%$ for elective and $11,1 \%$ for symptomatic cases ${ }^{13}$; although in some other reports, it reached $10 \%$ after elective repair. 14 The most frequent complications after elective OAR are cardiac and respiratory events along with wound infections, which were described to occur in the perioperative period in $10.2 \%, 7 \%$ and $3.5 \%$ of patients after surgery, respectively. ${ }^{13}$

Analysis of risk factors of early complications is the basis for the development of a strategy to effectively reduce the number of complications and increase the survival of patients after repairing surgery of AAA. There are limited data on this topic concerning the Polish population. Moreover, the importance of particular risk factors, like gender or diabetes, remain unclear. ${ }^{15,16}$

The aim of this study was to assess the type and frequency of early postoperative complications occurring in patients after surgical treatment of non-ruptured abdominal aortic aneurysms and to determine their risk factors.

\section{METHODOLOGY}

It was an observational study with medical records analyses of 205 patients operated for abdominal aortic aneurysm at the $4^{\text {th }}$ Military Teaching Hospital, Wroclaw, Poland, from January 2012 to December 2016. Patients with clinically and radiologically confirmed features of aneurysm rupture were excluded from the study. Complications and early deaths, defined as those occurring within 30 days of surgery, were recorded.

The data were collected from the digital medical documentation of consecutive patients, who were eligible according to inclusion criteria. Respiratory failure was defined as hypoxemia with or without hypercapnia in an arterial-blood gas test, circulatory failure as symptoms of peripheral hypoperfusion, hypotension (SBP $<90$ $\mathrm{mmHg}$ ) or pulmonary edema. Lower limb ischemia was defined as acute or critical ischemia, which required surgical intervention (including amputation). Kidney failure was defined as oliguria or anuria $(<0.5 \mathrm{ml} / \mathrm{kg} / \mathrm{h}$ for $6 \mathrm{~h})$ or serum creatinine increase ( $\geq 0.3 \mathrm{mg} / \mathrm{dl}$ within 48 $\mathrm{h}$ or $\geq 1.5$ times within 7 days). Wound infection was defined as clinical signs of local wound inflammation and positive culture from this site. Urinary tract infection was considered as positive urine bacterial culture and leukocytes count. Gastrointestinal bleeding was confirmed in endoscopy. Pulmonary embolism was confirmed with CT pulmonary angiography. Charac-teristics of the group were collected (demographic and clinical data, in particular concomitant diseases before the procedure and course of the surgery) and analysed to determine the risk factors for early complications.

Patients were qualified for elective surgery in accordance with the procedures contained in the current ESC guidelines for the diagnosis and treatment of the aortic diseases. ${ }^{11}$ Indications for the open surgical management were the aneurysm diameter greater than $55 \mathrm{~mm}$, fast growth dynamics (over $5 \mathrm{~mm} / 6$ months), atypical morphology, coexisting aneurysms of the iliac and popliteal artery. The indication for the elective surgery was the aforementioned aneurysm diameter, which is a threshold value for a significant increase of the risk of rupture. ${ }^{17,18}$

The operations were also carried out urgently in the case of symptomatic, unruptured aneurysms. In all patients, the aneurysm was located in the infrarenal aorta.

Exploratory analyses were carried out to determine risk factors for early complications. Complications were initially arranged together as the sum of observed in each patient, without their differentiation. Then, using logistic regression models, analyses of risk factors for the most common complications were performed. The significance level $\alpha=5 \%$ was established. During the quantitative analyses, Shapiro-Wilk's W test, Spearman's rank correlation and $U$ Mann-Whitney test were used. The following classification was used to estimate the strength of the $\mathrm{R}$ correlation: $<0.2$ - negligible or very weak correlation; 0.2-0.4 - weak; 0.4-0.7 - moderate; 0.7-0.9 strong; >0.9 - very strong. Changes in the blood parameters before and after the procedure were compared using Student's t-test for dependent groups. The regression model was built using the backward stepwise selection. The analyses were performed with Statistica (StatSoft, 13.0).

\section{RESULTS}

Men represented majority of the studied group ( $n=170$; $83 \%)$. The average age was $67.9 \pm 6.68$ years. Aneurysms $>55 \mathrm{~mm}$ were found in $110(53.6 \%)$ of patients. Extremely widened AAA (over $80 \mathrm{~mm}$ ) occurred in $22(10.7 \%$ of group). Symptomatic course was found in 54 patients $(26.3 \%)$ and in 5 patients $(2.4 \%)$, radiological dissection signs were observed.

In $132(64.4 \%)$ of patients, an aortic prostheses (tube grafts) were implanted, the others were provided with aorto-bifemoral (bifurcated) prostheses ( $n=73 ; 35.6 \%)$.

Hypertension ( $n=147 ; 71.7 \%)$ and coronary heart disease $(n=93 ; 45.3 \%)$ were the most common comorbidities. 
Dyslipidemia $(n=55 ; 26.8 \%)$, diabetes $(n=46 ; 22.4 \%)$, and a history of myocardial infarction $(n=39 ; 19.0 \%)$ were reported in patients with similar frequency. Peptic

Table I: Early complications in the open surgery management of nonruptured AAA $(n=205)$.

\begin{tabular}{lcc}
\hline Complications & Number of patients & Percentage (\%) \\
\hline Need for reoperation & 23 & 11.22 \\
Respiratory failure & 21 & 10.24 \\
Respiratory tract infection & 14 & 6.83 \\
Circulatory failure & 14 & 6.83 \\
Lower limb ischemia & 13 & 6.34 \\
Kidney failure & 12 & 5.85 \\
Death & 9 & 4.39 \\
Wound infection & 9 & 4.39 \\
Vascular prosthesis obstruction & 6 & 2.93 \\
Urinary tract infection & 3 & 1.46 \\
Gastrointestinal bleeding & 3 & 1.46 \\
Cardiac arrest & 3 & 1.46 \\
Bowels' ischemia & 3 & 1.46 \\
Lower limb amputation & 2 & 0.98 \\
Myocardial infarction & 2 & 0.98 \\
Pulmonary embolism & 2 & 0.98 \\
\hline
\end{tabular}

Table II: Associations between overall number of complications and selected factors.

\begin{tabular}{lcc}
\hline Total number of complications and: & $\begin{array}{c}\text { Correlation } \\
\text { coefficient R }\end{array}$ & $p$-value \\
\hline Duration of the surgery & 0.18 & 0.02 \\
Degree of blood loss & 0.33 & $<0.001$ \\
Age & - & 0.6 \\
Gender & - & 0.5 \\
Aneurysm diameter & - & 0.2 \\
Comorbidities & - & 0.1 \\
Overweight & - & 0.9 \\
Nicotinism & - & 0.1 \\
\hline
\end{tabular}

Table III: The most frequently observed complications and its established risk factors; OR - odds ratio.

\begin{tabular}{|c|c|}
\hline Complications & Predictors \\
\hline Reoperation necessity & $\begin{array}{l}\text { Aneurysm diameter (OR 1.02; } p=0.04 \text { ) } \\
\text { Blood loss (OR 4.8; } p<0.001) \\
\text { Surgery duration (OR 2.2; } p=0.015 \text { ) }\end{array}$ \\
\hline \multirow[t]{3}{*}{ Respiratory failure } & Aneurysm diameter (OR 1.03; $p=0.01$ ) \\
\hline & Blood loss (OR 3.7; p=0.002) \\
\hline & Surgery duration (OR 2.2; $p=0.015$ ) \\
\hline \multirow[t]{5}{*}{ Circulatory failure } & Patient's age (OR 1.1; $p=0.035$ ) \\
\hline & Aneurysm diameter (OR 1.04; $p=0.002)$ \\
\hline & Blood loss (OR 6.4; $p<0.001$ ) \\
\hline & Surgery duration (OR 2.8; $p=0.009$ ) \\
\hline & Diabetes (OR 2.3; $p=0.003)$ \\
\hline \multirow[t]{3}{*}{ Lower limb ischaemia } & Surgery duration (OR 1.99; $p=0.048$ ) \\
\hline & Blood loss (OR 3.7; p=0.01) \\
\hline & BMI (OR 2.6; $p=0.03)$ \\
\hline \multirow[t]{4}{*}{ Kidney failure } & Aneurysm diameter (OR 1.03; $p=0.02$ ) \\
\hline & Blood loss (OR 2.8; p=0.046) \\
\hline & Surgery duration (OR $2.9 ; p=0.009$ ) \\
\hline & Diabetes $(\mathrm{OR} 2.4 ; \mathrm{p}=0.005)$ \\
\hline \multirow[t]{4}{*}{ Death } & Aneurysm diameter (OR 1.04; $p=0.003)$ \\
\hline & Surgery duration (OR $2.69 ; p=0.02)$ \\
\hline & Diabetes (OR 2.17; p=0.025) \\
\hline & Blood loss (OR 2.99; $p=0.06$ ) \\
\hline
\end{tabular}

ulcer $(n=21 ; 10.2 \%)$, carotid artery stenosis $(n=20$; $9.7 \%)$, and chronic heart failure $(n=19 ; 9.2 \%)$ occurred less frequently. The majority of patients presented a history of smoking $(n=144 ; 70.2 \%)$ and $B M I>25$ in a physical examination $(n=130 ; 63.4 \%)$.

Patients required an average transfusion of 2.9 packed red blood cells units (95\% Cl=2.62-3.19). In 124 (60.5\%) of patients, the amount of blood loss during the treatment ranged from $500 \mathrm{ml}$ to $1000 \mathrm{ml}$. Loss above $1000 \mathrm{ml}$ was observed in $46(22.4 \%)$. The number of patients who lost less than $500 \mathrm{ml}$ of blood was $35(17.07 \%)$. The average duration of the procedure was 1.88 hours $(95 \% \mathrm{Cl}=1.78$ 1.99). The average hospitalisation length was about 10 days $(95 \% \mathrm{Cl}$ 9.41-11.37).

The most common complication observed up to 30 days after surgery was the need for a reoperation (Table I). Twenty-three patients required reintervention. In 14 of them, it was performed once; in seven cases, there had to be two additional surgeries, and two patients required three re-operations. The reason for performing such a procedure was most often sudden deterioration of the patient's condition, including hypovolemic shock.

At first, the complications were analysed without its differentiation, just as its overall number. The associations are presented in Table II.

Univariate logistic regression was used to determine the predictors for the most frequently observed complications, which were analysed there separately. The results were shown in Table III.

\section{DISCUSSION}

Early complications after OAR treatment of unruptured abdominal aortic aneurysms in our study have a similar qualitative and quantitative structure to complications occurring in other centres.17-23 The percentage of necessary reinterventions observed in our study, at the level of $11.2 \%$, was higher than reported by other authors. It ranged, depending on the study, from $0.6 \%$ to $7.1 \% .22,23$ This difference may result from a greater burden of comorbidities, especially cardiovascular diseases and the qualification of patients with larger diameter aneurysms. 10,21-23 Independent risk factors for re-intervention present in the literature are: age, diameter of the aneurysm, renal failure and ischemic heart disease.19,23 Predictors, not included in other studies, and increasing the percentage of re-intervention, which were found in our analysis, comprise the amount of blood lost during surgery and the surgery duration.

Respiratory complications occurred as the most frequent. Respiratory failure in a postoperative period was diagnosed in $10.24 \%$ of these patients. It is one of the most common, and at the same time, one of the most serious complications, closely related to the invasiveness of surgery. ${ }^{19,21}$ Cardiovascular compli-cations, such as 
circulatory failure, ischemia of the lower limb or myocardial infarction, are also frequent. Similar complication rate was reported previously by other authors. In the group of patients with nonruptured infrarenal AAA analysed by Law et al., cardiac events occurred in $10.2 \%$ and respiratory events in $7 \%$ of them within 30 days after repair. ${ }^{13}$ Significantly higher rate of pulmonary complications, reaching $42.2 \%$ and observed by Dayama et al., may be due to the fact that other localisations of aneurysm were also included in the studied group. ${ }^{14}$ There are publications that associate OAR with an increased risk of deterioration of renal function and the development of kidney failure. In the analysed group, $5.85 \%$ of patients developed it. This is similar or slightly lower than convergent studies. ${ }^{19-21}$

Perioperative mortality (30 days) presented in other centres did not exceed $4.5 \%{ }^{18}$, which is similar to the results of our analyses. The low percentage of deaths found after OAR may be associated with the experience of surgeons in our clinic, proper risk assessment, and prudential qualification of patients with an appropriately high chance of survival. Regression analyses showed the effect of the aneurysm diameter, the duration of the procedure, the degree of blood loss, and diabetes on the increased mortality. However, coronary heart disease and renal failure did not occur as risk factors for death, as reported in other papers. ${ }^{19,23}$

Age remains a controversial parameter used to assess the risk of postoperative complications after OAR. Statistical tests carried out showed no significant correlations between age and the total number of complications. However, it was connected with the higher incidence of circulatory failure. Other authors describe a significant relationship between age and the complications incidence, particularly mortality, in the case of both ruptured and unruptured aneurysms. ${ }^{19,24}$ Age is an important parameter of the Glasgow aneurysm score. GAS scale is a useful tool for efficient postoperative complications risk stratification. ${ }^{19}$ GAS has a high negative predictive value for low-risk patients, as opposed to high-risk ones. ${ }^{25}$ For this reason, it should be used to determine the predictive values of the occurrence of early postoperative complications after OAR in patients of the younger age group with a lower burden of comorbidities. ${ }^{25}$

There are several limitations of this study. At first, the data is retrospective, which might impair the precision of study design, its results and established conclusions. Secondly, the numbers of respective complications are relatively low, resulting in a bias while performing multivariate analysis, which would establish the actual associations and risk factors more accurate. That is the reason why only univariate logistic regression was used.

\section{CONCLUSION}

Intraoperative blood loss, longer surgery time, and larger diameter of the aneurysm are the risk factors for early complications. Increased body weight also seems to adversely affect the postoperative course. There is a need to shorten the duration of the procedure and minimise blood loss.

\section{ETHICAL APPROVAL:}

The study was approved by the Commission of Bioethics at Wroclaw Medical University, Wroclaw, Poland.

\section{PATIENTS' CONSENT:}

Written informed consent in Polish was obtained from all patients for the publication of this paper.

\section{CONFLICT OF INTEREST:}

Authors declared no conflict of interest.

\section{AUTHORS' CONTRIBUTION:}

DJ, KM: Helped to draft the manuscript.

WB: Participated in the design of the study.

$\mathrm{KB}, \mathrm{MM}$ : Participated in the design and coordination of the paper.

PM: Authored the manuscript.

MC: Arranged the manuscript.

\section{REFERENCES}

1. Kent KC. Clinical practice. Abdominal aortic aneurysms. N Engl J Med 2014; 371:2101-8.

2. Ashton HA, Buxton MJ, Day NE, Kim LG, Marteau TM, Scott RA, et al. The Multicentre Aneurysm Screening Study (MASS) into the effect of abdominal aortic aneurysm screening on mortality in men: A randomised controlled trial. Lancet 2002; 360:1531-9.

3. Norman PE, Jamrozik K, Lawrence-Brown MM, Le MT, Spencer CA, Tuohy RJ, et al. Population-based randomised controlled trial on impact of screening on mortality from abdominal aortic aneurysm. BMJ 2004; 329:1259.

4. Crawford ES, Cohen ES. Aortic aneurysm: A multifocal disease. Presidential address. Arch Surg 1982; 117:1393-400.

5. Larsson E, Vishnevskaya L, Kalin B, Granath F, Swedenborg J, Hultgren R. High frequency of thoracic aneurysms in patients with abdominal aortic aneurysms. Ann Surg 2011; 253:180-4.

6. Chaikof EL, Brewster DC, Dalman RL, Makaroun MS, Illig KA, Sicard GA, et al. SVS practice guidelines for the care of patients with an abdominal aortic aneurysm: Executive summary. J Vasc Surg 2009; 50:880-96.

7. Moll FL, Powell JT, Fraedrich G, Verzini F, Haulon S, Waltham M, et al. Management of abdominal aortic aneurysms clinical practice guidelines of the European society for vascular surgery. Eur J Vasc Endovasc Surg 2011; 41(Suppl 1):S1-S58.

8. van de Luijtgaarden KM, Heijsman D, Maugeri A, Weiss MM, Verhagen $\mathrm{HJ}$, IJpma A, et al. First genetic analysis of aneurysm genes in familial and sporadic abdominal aortic aneurysm. Hum Genet 2015; 134:881-93.

9. Hagerty T, Geraghty P, Braverman AC. Abdominal aortic aneurysm in Marfan syndrome. Ann Vasc Surg 2017; 40: 294.e1-e6.

10. Lederle FA, Wilson SE, Johnson GR, Reinke DB. Immediate repair compared with surveillance of small abdominal aorta aneurysms. N Engl J Med 2002; 346:1437-44. 
11. Ali MU, Fitzpatrick-Lewis D, Miller J, Warren R, Kenny M, Sherifali $\mathrm{D}$, et al. Screening for abdominal aortic aneurysm in asymptomatic adults. $J$ Vasc Surg 2016; 64:1855-68.

12. Takagi H, Matsui M, Umemoto T. A meta-analysis of clinical studies of statins for prevention of abdominal aortic aneurysm expansion. J Vasc Surg 2010; 52:1675-81.

13. Law Y, Chan Y, Cheung G, Ting A, Cheng S. Outcome and risk factor analysis of patients who underwent open infrarenal aortic aneurysm repair. Asian J Surg 2016; 39:164-71.

14. Dayama A, Sugano D, Reeves J, Rivera A, Tsilimparis N. Early outcomes and perioperative risk assessment in elective open thoracoabdominal aortic aneurysm repair: An analysis of national data over a five-year period. Vascular 2015; 24:3-8.

15. de Guerre LEVM, Varkevisser RRB, Swerdlow NJ, Liang P, Li C, Dansey $\mathrm{K}$, et al. Sex differences in perioperative outcomes after complex abdominal aortic aneurysm repair. $J$ Vasc Surg 2019; 31229-7.

16. Hughes K, Jackson J, Prendergast T, Rose D, Bolorunduro O, Obirieze A, et al. Diabetes mellitus is not associated with major morbidity following open abdominal aortic aneurysm repair. J Surg Res 2013; 184:751-4.

17. Dillavou ED, Muluk SC, Makaroun MS. A decade of change in abdominal aortic aneurysm repair in the United States: Have we improved outcomes equally between men and women? J Vasc Surg 2006; 43:230-8

18. McPhee JT, Hill JS, Eslami MH. The impact of gender on presentation, therapy, and mortality of abdominal aortic aneurysm in the United States, 2001-2004. J Vasc Surg 2007; 45:891-9.
19. Biancari F, Leo E, Ylönen K, Vaarala MH, Rainio P, Juvonen T. Value of the Glasgow aneurysm score in predicting the immediate and long-term outcome after elective open repair of infrarenal abdominal aortic aneurysm. Br J Surg 2003; 90: 838-44.

20. Chiesa R, Tshomba Y, Psacharopulo D, Rinaldi E, Logaldo D, Marone EM, et al. Open repair for infrarenal AAA: Technical aspects. J Cardiovasc Surg (Torino) 2012; 53(1 Suppl 1): 119-31.

21. Dakour Aridi HN, Locham S, Nejim B, Ghajar NS, Alshaikh H, Malas MB. Indications, risk factors, and outcomes of 30-day readmission after infrarenal abdominal aneurysm repair. J Vasc Surg 2018; 67:747-758.e7.

22. Zabrocki L, Marquardt F, Albrecht K, Kribben A, HergetRosenthal S. Permanent decline of renal function after infrarenal abdominal aortic aneurysm repair - frequency and risk factors. Ann Vasc Surg 2018; 47:272-8.

23. Deery SE, O'Donnell TFX, Bodewes TCF, Dalebout BA, Pothof AB, Shean $\mathrm{KE}$, et al. Early reintervention after open and endovascular abdominal aortic aneurysm repair is associated with high mortality. J Vasc Surg 2018; 67:433-440.e1.

24. Dimick JB, Stanley JC, Axelrod DA, Kazmers A, Henke PK, Jacobs LA, et al. Variation in death rate after abdominal aortic aneurysmectomy in the United States. Ann Surg 2002; 235: 579-85.

25. Hirzalla O, Emous M, Ubbink DT, Legemate D. External validation of the Glasgow aneurysm score to predict outcome in elective open abdominal aortic aneurysm repair. $J$ Vasc Surg 2006; 44:712-6. 